\title{
Influences of multiple spatial stimulus and response codes on orthogonal stimulus-response compatibility
}

\author{
YANG SEOK CHO and ROBERT W. PROCTOR \\ Purdue University, West Lafayette, Indiana
}

\begin{abstract}
When up-down stimuli are mapped to left-right responses, an up-right/down-left mapping advantage is found that is modified by response eccentricity and hand posture. These effects can be attributed to correspondence of asymmetric stimulus and response codes formed relative to multiple reference frames. We examined the influence of stimulus-set location on these orthogonal stimulus-response compatibility (SRC) effects. In Experiment 1, the stimulus set appeared in the upper or lower display positions. A spatial code for stimulus-set location was formed, producing Simon-type response eccentricity and hand posture effects, but this code had no influence on the coding of the relevant stimuli. In Experiment 2, the stimulus set appeared in the left, center, or right positions relative to the response location, which also varied, to dissociate the effects of response location, relative to the stimulus display and body midline. The former factor influenced the orthogonal SRC effect for both unimanual switch movements and bimanual keypresses, and the latter factor influenced the effect for only unimanual switch movements. Stimulus-set location causes orthogonal Simon-type effects when varied along the stimulus dimension and provides a referent for response coding when varied along the response dimension.
\end{abstract}

In spatial choice-reaction tasks, performance is better when stimulus locations are assigned to their spatially corresponding responses than when they are not (see Proctor \& Reeve, 1990). This effect of the spatial relation between stimuli and responses is called a stimulus-response compatibility (SRC) effect. The SRC effect also occurs when the location of the stimulus is irrelevant to selection of the correct response (see Hommel \& Prinz, 1997), a phenomenon called the Simon effect. Many researchers attribute both SRC proper and the Simon effect to differences in the time required for response selection to occur. Specifically, response selection is considered to be faster when the spatial code for the stimulus corresponds to the spatial code for the response than when it does not (e.g., Lu \& Proctor, 1995).

Stimulus codes are formed relative to different reference frames (or objects), and when multiple reference frames are available, the overall RT reflects the summed contribution of these codes (e.g., Lamberts, Tavernier, \& d'Ydewalle, 1992; Roswarski \& Proctor, 1996). For example, in Lamberts et al.'s Experiment 2, the stimulus could occur in one of eight locations, ordered left to right, that varied with respect to hemispace (the four leftmost vs. four rightmost locations), hemifield within hemispace, and rel-

\footnotetext{
We thank anonymous reviewers for helpful comments on a previous version of this article. We also thank Leigh Lytle, Srikanth Ramachandran, and Kerry Thorne for their assistance with data collection. Correspondence should be addressed to Y. S. Cho, Department of Psychological Sciences, Purdue University, West Lafayette, IN 47907-2004 (e-mail: yscho@psych.purdue.edu).
}

ative position within hemifield. All of these reference frames contributed to the Simon effect in an additive manner. That is, the Simon effect was largest for the extreme left and right stimulus locations, for which the location codes were the same, relative to all three reference frames.

\section{Up-Right/Down-Left Advantage}

The mapping of stimuli to responses also affects performance when the stimulus and response sets vary along orthogonal dimensions (e.g., Bauer \& Miller, 1982). With a vertical stimulus set mapped to a horizontal response set, the up-right/down-left mapping yields better performance than does the up-left/down-right mapping, regardless of whether responses are unimanual movements, bimanual keypresses, or vocal "left"-"right" utterances (e.g., Weeks \& Proctor, 1990). This effect is called the overall upright/down-left advantage, and researchers have generally agreed (e.g., Adam, Boon, Paas, \& Umiltà, 1998; Cho \& Proctor, 2003; Lippa \& Adam, 2001) that it is due to correspondence of asymmetric stimulus and response codes, as suggested originally by Weeks and Proctor's salient features coding account.

According to this account, stimuli and responses are coded asymmetrically on their respective dimensions, with the spatial codes for up and right typically being more "salient" (i.e., of positive polarity) than those for down and left (which are of negative polarity), respectively (Chase \& Clark, 1971; Just \& Carpenter, 1975; Seymour, 1974). Performance is better when the asymmetric structure of the stimulus set is maintained in the mapping to the response set than when it is not (Proctor, 
Reeve, \& Van Zandt, 1992). That is, responding is faster with the up-right/down-left mapping because the two positive codes correspond, as do the two negative codes, whereas for the up-left/down-right mapping they do not.

\section{Response Eccentricity and Hand Posture Effects}

For unimanual responses, the orthogonal SRC effect is influenced by the position at which the responses are made (e.g., Michaels, 1989). The up-right/down-left advantage obtained at the center position is amplified when responses are made in the right hemispace but reversed to an up-left/down-right advantage when responses are made in the left hemispace, regardless of whether the left or right hand is used for responding (e.g., Weeks, Proctor, \& Beyak, 1995). Also, orthogonal SRC is affected by hand posture (e.g., Michaels \& Schilder, 1991). When the hand is in the typical prone posture, the up-right/downleft advantage is usually larger with the left hand than with the right hand, whereas when the hand is in a supine posture, the right hand shows a larger up-right/down-left advantage than does the left hand.

Lippa and Adam (2001) accepted that the up-right/ down-left advantage is due to correspondence of asymmetric stimulus and response codes, but concluded that the effects of response eccentricity and hand posture on orthogonal SRC require a different type of explanation. They proposed an end-state comfort hypothesis, according to which the response dimension is mentally rotated $90^{\circ}$ to align it with the stimulus dimension, with the rotation being in the direction that would result in the most comfortable end state if the hand were actually rotated. According to Lippa and Adam, with a prone hand posture, both hands prefer a counterclockwise rotation in the right hemispace and a clockwise rotation in the left hemispace. Consequently, the two hands show similar effects of response position: an up-right/down-left advantage in the right hemispace and an up-left/down-right advantage in the left hemispace.

However, a series of experiments by Proctor and Cho (2003; Cho \& Proctor, 2002) provided evidence that is inconsistent with the end-state comfort hypothesis. In Proctor and Cho's (2003) Experiment 1, the response eccentricity effect was obtained with keypresses executed with the left and right index fingers. When subjects made responses at three positions, the up-right/down-left advantage found at body midline increased in the right hemispace and reversed to an up-left/down-right advantage in the left hemispace. The magnitude of this response eccentricity effect was similar to that obtained with unimanual movement responses. This outcome implies that the response eccentricity effect is due to properties of central processing that do not depend on a specific response mode, which is in contrast to the emphasis that the endstate comfort hypothesis places on motoric properties of the responding hand.

Because of this emphasis, the end-state comfort hypothesis suggests that the orthogonal SRC effect is determined by hand position relative to body midline. However,
Weeks et al. (1995) showed the importance of the relative location at which the responses are made. In their Experiment 2, the position of the response switch relative to an inactive switch was manipulated, with the responding hand always placed at body midline. Orthogonal SRC was affected by the location of the inactive response switch, with the advantage for the up-right/down-left mapping being larger when the response position was to the right of the inactive switch than when it was to the left. Proctor and Cho (2003) replicated this relative response-location effect with left-right joystick movements and left-right keypresses. These results imply that the response eccentricity effect is not a consequence of egocentric hand position.

In Cho and Proctor's (2002) Experiment 1, subjects made left-right toggle-switch movements at three response positions in two hand postures, prone and supine. Response position and hand posture affected the orthogonal SRC effect in an additive manner. This result pattern is counter to the implication of the end-state comfort hypothesis that the orthogonal SRC effect is due to interplay of the responding hand and hand position. Moreover, Cho and Proctor (2002) showed in their Experiment 2 that the hand posture effect obtained by grasping with the thumb and index finger was significantly decreased when subjects grasped a toggle switch with their ring and small fingers. The results of the two experiments imply that the hand posture effect is due at least in part to the coding of response position, and that multiple spatial codes for the response position are formed relative to available reference frames, with their effects combining in a relatively independent manner. In sum, the findings suggest that the orthogonal mapping preference is affected by response position relative to multiple reference frames, and that the response eccentricity and hand posture effects are due to properties of central processing, rather than to motoric properties of a specific response limb.

\section{A Multiple Asymmetric Codes Account}

Although the findings described in the previous section are difficult to reconcile with the end-state comfort hypothesis, they can be accommodated by an asymmetric coding account of the type used to explain the overall upright/down-left advantage. For the response eccentricity effect, Weeks et al. (1995) proposed that response location is coded, and that the code for the response alternative consistent with the response-location code becomes the most salient. Consequently, the right response code is salient for responding in the right hemispace, resulting in a large upright/down-left advantage. In contrast, the left response code is salient for responding in the left hemispace, resulting in a shift to an up-left/down-right advantage.

Cho and Proctor (2003) elaborated this position in what we will call the multiple asymmetric codes account. One fundamental tenet of this account is that spatial codes for the response location are formed relative to available reference frames, including the stimulus display (Cho \& Proctor, in press-a), another response apparatus (Proctor \& Cho, 2003; Weeks et al., 1995), and the main part of 
the responding hand (Cho \& Proctor, 2002). The other fundamental tenet of this account is correspondence of asymmetric codes, as specified in Weeks and Proctor's (1990) salient features coding account: Within each reference frame, the polarity is positive for the response code that is consistent with the representation of the response position as left or right, and the polarity is negative for the response code that is inconsistent with this representation. The correspondences of these polar spatial codes with those of the stimulus dimension (positive for up and negative for down) contribute to the overall SRC effect in an approximately additive manner.

This account in terms of multiple asymmetric codes is in agreement with the general view, described in the introductory paragraphs of this article, that response selection is influenced by multiple spatial codes formed relative to available reference frames. Notably, effects of asymmetric codes on orthogonal stimulus-response ( $\mathrm{S}-\mathrm{R}$ ) dimensions can be present simultaneously with those due to correspondence of codes on parallel dimensions. Hommel and Lippa (1995) had subjects make left-right keypresses to up or down stimuli presented in the context of a picture of Marilyn Monroe's face, tilted $90^{\circ}$ to the left or right, such that the stimulus locations were the left and right eyes. They found a 7-msec eye-response correspondence effect, indicating that stimulus locations were coded as left or right in terms of the face, as well as a 28-msec up-right/down-left advantage in the mean RT data, indicating that the stimulus locations were also coded up or down. Similarly, in a follow-up experiment, Proctor and Pick (1999, Experiment 1B) found an 18-msec eye-response correspondence effect and a 42-msec upright/down-left advantage when subjects were not allowed to move or tilt their heads by using a chin/forehead rest.

\section{Effects of Stimulus-Set Location}

Most studies of the response eccentricity and hand posture effects on orthogonal SRC have manipulated the location of the response set and other response-related variables. However, according to the multiple asymmetric codes account, manipulations of stimulus-set, or display location should also affect performance. We recently reported experiments, as will be described in detail in the introductions to Experiments 1 and 2 of the present study, in which stimulus-set location was varied along the same dimension as the stimuli (Cho \& Proctor, in press-b) or the responses (Cho \& Proctor, in press-a). When varied along the stimulus dimension, stimulus-set location had no influence on the orthogonal SRC effect, but when varied along the response dimension, it did. However, the pattern of results suggested that this effect was a consequence of coding the response-set position relative to the stimulus set. Together, the results of these experiments imply that stimulus-set location has no effect on the asymmetry of the stimulus codes.

To minimize the complexity of their experiments, Cho and Proctor (in press-a, in press-b) did not vary response position factorially with stimulus-set location. Because of this procedural restriction, the results were ambiguous regarding several important questions that cannot be answered without a complete factorial design. When stimulusset location varies along the same dimension as the stimuli, is stimulus-set location even coded? Obtaining response eccentricity and hand posture effects for stimulus-set location would provide an affirmative answer to this question. Moreover, it would provide evidence that a Simon-type effect for irrelevant location information can occur across orthogonal S-R dimensions. Will any such effects of response eccentricity and hand posture for stimulus-set location be additive, as the multiple asymmetric codes account suggests? Does this coding of stimulus-set location have a significant effect on orthogonal SRC when responding in the right or left hemispace, response positions that typically yield larger orthogonal SRC effects than does body midline position? Experiment 1 answered these questions with factorial manipulations of stimulus-set location along the vertical dimension on which stimulus location varied, response position along the horizontal dimension on which the responses varied, the hand used for responding, and hand posture (prone or supine).

When stimulus-set location varies along the same dimension as the responses, is performance influenced by coding hand position relative to body midline, for which there was a nonsignificant tendency in Cho and Proctor's (in press-a) study, as well as by coding response location relative to the display? Does this effect of spatial coding for hand position occur only for unimanual responses, and does it combine additively with the effect of spatial coding for response-set location, as predicted by the multiple asymmetric codes account? Experiment 2 was designed to answer these questions by including factorial manipulations of response position, stimulus-set location along the horizontal dimension on which response position varied, and response mode (unimanual left hand, unimanual right hand, bimanual keypress).

\section{EXPERIMENT 1}

Cho and Proctor (in press-b) reported two experiments in which stimulus-set location was varied along the same dimension as that of the stimulus alternatives, with responses made on the orthogonal dimension at body midline. In their Experiment 1, up-down stimuli were mapped to left-right responses, and the stimulus set appeared in the upper or lower half of the display screen. An up-right/ down-left advantage occurred, and stimulus-set location had no influence on this orthogonal SRC effect. To rule out the possibility that the lack of influence was due to inflexibility of coding along the vertical dimension (e.g., Clark, 1973), left-right stimuli were mapped to up-down responses in Experiment 2, and the stimulus set appeared in the left or right half of the display screen. This experiment also showed no effect of stimulus-set location on orthogonal SRC, suggesting that stimulus-set location does not alter the asymmetry of the stimulus codes for either the vertical or horizontal dimension. 
The lack of effect of stimulus-set location on the asymmetry of the stimulus codes would be relatively uninteresting if it were due simply to stimulus-set location not being coded. The Simon effect is typically interpreted as showing that irrelevant stimulus-location information is coded automatically (see, e.g., Lu \& Proctor, 1995). However, in the Simon task, the stimuli vary along the same spatial dimension (horizontal) as the responses, which may "prime" location on this dimension to be coded and processed. Consequently, one could still argue that the lack of influence of stimulus-set location in Cho and Proctor's (in press-b) experiments was due to its not being coded, rather than to its representation not affecting the coding of the relevant stimuli.

One way to establish that stimulus-set location is in fact coded is to show an orthogonal Simon-type effectthat is, faster right responses when the stimulus set is in the upper position and faster left responses when it is in the lower position - but no such effect was evident in Cho and Proctor's (in press-b) study. A stronger test for coding of stimulus-set location can be made by varying response eccentricity and hand posture, and seeing whether these manipulations produce Simon-type effects similar to the effects they produce on orthogonal SRC. Significant response eccentricity and hand posture effects with respect to stimulus-set location would provide evidence that stimulus-set location was indeed coded. Moreover, according to the multiple asymmetric codes account, the response and hand posture effects should not interact.

Although stimulus-set location did not affect orthogonal SRC in Cho and Proctor's (in press-b) study in which responses were made at body midline, it could influence orthogonal SRC when responses are made in the right or left hemispace, where the orthogonal SRC effects are typically larger. However, the multiple asymmetric codes account predicts that an interaction of this type should not occur. Any influence of stimulus-set location on orthogonal SRC should be independent of the effects of response eccentricity and hand posture.

To examine these issues, response-set location and prone versus supine hand posture were varied along with stimulus-set location in Experiment 1. The stimuli were up-down locations mapped to unimanual left-right responses made with prone and supine hand postures at body midline, left of midline, or right of midline, and the stimulus set could appear in either the upper or the lower half of the display screen. If the irrelevant stimulus-set location is coded, a Simon-type effect should occur, with RT shorter when the polarity, or salience, of the code for stimulus-set location corresponds with that of the response than when it does not. If, in addition, the code for stimulusset location affects the polarity of the up-down stimulus codes similar to how response-set location affects the polarity of the response codes, an up-right/down-left advantage should be obtained when the stimulus set is in the upper position, and an up-left/down-right advantage should be obtained when it is in the lower position.

\section{Method}

Subjects. Forty-eight undergraduates enrolled in introductory psychology classes at Purdue University participated in partial fulfillment of a course requirement. All were right-handed and reported normal or corrected-to-normal visual acuity. Subjects were randomly assigned to two groups, one for which responses were made with the left hand and the other for which responses were made with the right hand.

Stimuli and Apparatus. Micro Experimental Laboratory 2 (MEL 2.01) software was used to program the experiment. Stimuli were presented on the display screen of an IBM-compatible microcomputer. Responses were made by deflecting a toggle switch left or right in response to the spatial location of the imperative stimulus. The toggle switch was mounted on a panel $(43 \times 17.5 \times 6 \mathrm{~cm})$ interfaced with a MEL 2 response box. The height of the toggle switch was $7.5 \mathrm{~cm}$. Half the subjects in each response-hand group were asked to hold the switch in a palm-down posture (prone), and the other half in a palm-up posture (supine). Viewing distance was approximately $60 \mathrm{~cm}$. Stimuli were standard uppercase Xs $(0.3 \times 0.4 \mathrm{~cm}$, approximately $0.29^{\circ} \times 0.39^{\circ}$ of visual angle) and were presented as white characters on a dark background, approximately $2 \mathrm{~cm}\left(1.91^{\circ}\right)$ above or below a fixation row " +++ " $\left(0.9 \times 0.3 \mathrm{~cm}, 0.86^{\circ} \times 0.29^{\circ}\right)$.

Procedure. Subjects were asked to align their body midline with the center of the screen. Responses were made at three different locations: $20 \mathrm{~cm}$ left of body midline, body midline, and $20 \mathrm{~cm}$ right of body midline. For both hand postures, the toggle switch was held between the thumb and index finger, using the thumb to hold the switch against the finger between the knuckle and the fingertip (see Figure 1). The experiment consisted of two three-block sessions with a 2-min resting period between them. The up-right/down-left mapping was used for one session and the up-left/down-right mapping for the other session, with the order of the mappings counterbalanced across subjects. Half the subjects began at the $20-\mathrm{cm}$ position in the right hemispace and progressed to the left; the other half began at the $20-\mathrm{cm}$ position in the left hemispace and progressed to the right. Each subject performed 30 practice trials when the new mapping was introduced, and each block consisted of 100 trials. A 30-sec rest period was given after completion of each block.

At the beginning of each trial, a fixation asterisk $(0.2 \times 0.2 \mathrm{~cm}$, $\left.0.19^{\circ} \times 0.19^{\circ}\right)$ was presented at the center of the screen. Subjects were asked to stare at it. After $250 \mathrm{msec}$, the asterisk disappeared, and the fixation row of three plus signs was presented either $4.5 \mathrm{~cm}\left(4.30^{\circ}\right)$ above or below the fixation asterisk. After another $250 \mathrm{msec}$, an X was presented as the imperative stimulus above or below the fixation row, both of which remained on until the subject responded. The fixation asterisk for the next trial came on $1 \mathrm{sec}$ after the response or, when the response was incorrect, after a 500-msec feedback tone.

\section{Results}

RTs shorter than $125 \mathrm{msec}$ and longer than $1,250 \mathrm{msec}$ were removed, with $1.27 \%$ of the trials excluded. Mean RT and percent error (PE) were calculated for each subject as a function of mapping (up-right/down-left, upleft/down-right), stimulus-set location (upper, lower), response-set location (left, center, right), and response (left, right). Analyses of variance (ANOVAs) were conducted on the RT and PE data, with those variables as within-subjects factors, and response hand (left, right) and hand posture (prone, supine) as between-subjects factors (see Table 1, which collapses across responses).

Overall mapping effect. Consistent with previous studies, an overall up-right/down-left mapping advantage was obtained: Responses were faster and PE lower 


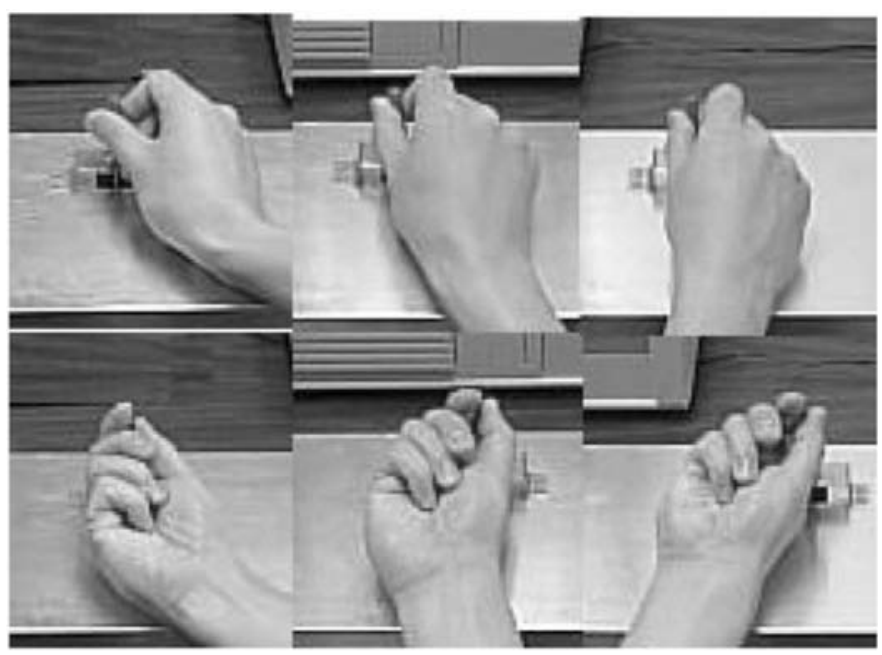

Figure 1. Hand postures used in Experiment 1, illustrated for the right hand. The top row shows the prone posture, and the bottom row the supine posture, in the left, center, and right response-set positions.

for the up-right/down-left mapping $(M \mathrm{~s}=523 \mathrm{msec}$ and $3.36 \%$, respectively) than for the up-left/down-right mapping $(M \mathrm{~s}=543 \mathrm{msec}$ and $4.47 \%)[F \mathrm{~s}(1,44)=4.66$ and $4.65, M S_{\mathrm{e}} \mathrm{s}=26,011$ and $\left.76.29, p \mathrm{~s}=.036\right]$.

The PE data showed two relatively unimportant interactions involving mapping that the RT data did not: an interaction of mapping with response $[F(1,44)=5.43$, $\left.M S_{\mathrm{e}}=18.05, p=.0244\right]$ and a three-way interaction of these variables with stimulus-set location $[F(1,44)=$ 4.29, $\left.M S_{\mathrm{e}}=27.89, p=.0441\right]$. For the upper stimulus set, the right response showed a $1.91 \%$ up-right/downleft advantage, whereas the left response showed a $0.54 \%$ up-left/down-right advantage. However, for the lower stimulus set, the right and left responses showed similar magnitudes of the up-right/down-left advantage $(1.47 \%$ and $1.60 \%$ for the right and left responses, respectively). Another way of describing this pattern is that for the upper stimulus set, responses were more accurate to the up stimulus than to the down stimulus, but for the lower stimulus set, there was little difference between the two stimulus alternatives.

Hand posture effect. The hand posture effect refers to the combined influence of the variables response hand and hand posture on the mapping effect. Thus, it should be evident as a three-way interaction of these variables. In fact, for the RT data, the three-way interaction of mapping, response hand, and hand posture was significant $\left[F(1,44)=6.59, M S_{\mathrm{e}}=26,011, p=.0137\right]$, even though mapping did not interact separately with either response hand or hand posture $(F \mathrm{~s}<1.0)$. As in Cho and Proctor's

Table 1

Mean Reaction Times (RTs, in Milliseconds) and Percentages of Error (PEs) in Experiment 1 as a Function of Mapping, Response-Set Location (Left, Center, Right), Response Hand, Stimulus-Set Location (Upper, Lower), and Hand Posture

\begin{tabular}{|c|c|c|c|c|c|c|c|c|c|c|c|c|}
\hline \multirow[b]{3}{*}{ Hand and Mapping } & \multicolumn{4}{|c|}{ Left } & \multicolumn{4}{|c|}{ Center } & \multicolumn{4}{|c|}{ Right } \\
\hline & \multicolumn{2}{|c|}{ Upper } & \multicolumn{2}{|c|}{ Lower } & \multicolumn{2}{|c|}{ Upper } & \multicolumn{2}{|c|}{ Lower } & \multicolumn{2}{|c|}{ Upper } & \multicolumn{2}{|c|}{ Lower } \\
\hline & RT & $\mathrm{PE}$ & RT & $\mathrm{PE}$ & RT & $\mathrm{PE}$ & RT & $\mathrm{PE}$ & RT & $\mathrm{PE}$ & RT & $\mathrm{PE}$ \\
\hline \multicolumn{13}{|c|}{ Prone Posture } \\
\hline Left Hand & & & & & & & & & & & & \\
\hline Up-right/down-left & 549 & 4.09 & 548 & 4.15 & 531 & 3.46 & 526 & 2.73 & 528 & 1.63 & 527 & 1.38 \\
\hline Up-left/down-right & 567 & 4.73 & 572 & 3.48 & 593 & 3.51 & 588 & 5.85 & 597 & 4.14 & 592 & 3.91 \\
\hline \multicolumn{13}{|l|}{ Right Hand } \\
\hline Up-right/down-left & 557 & 10.6 & 548 & 7.33 & 511 & 5.09 & 501 & 5.71 & 500 & 4.25 & 503 & 5.45 \\
\hline Up-left/down-right & 516 & 1.80 & 506 & 4.32 & 539 & 6.87 & 538 & 7.36 & 537 & 7.17 & 533 & 6.4 \\
\hline \multicolumn{13}{|c|}{ Supine Posture } \\
\hline Left Hand & & & & & & & & & & & & \\
\hline Up-right/down-left & 554 & 3.55 & 533 & 3.51 & 508 & 1.78 & 503 & 1.69 & 493 & 1.01 & 493 & 1.44 \\
\hline Up-left/down-right & 480 & 2.83 & 465 & 3.34 & 502 & 4.39 & 502 & 3.77 & 524 & 3.81 & 517 & 4.92 \\
\hline \multicolumn{13}{|l|}{ Right Hand } \\
\hline Up-right/down-left & 548 & 2.48 & 550 & 2.06 & 515 & 2.02 & 500 & 0.65 & 507 & 2.17 & 511 & 2.54 \\
\hline Up-left/down-right & 551 & 2.21 & 549 & 2.20 & 564 & 4.22 & 548 & 5.27 & 584 & 4.76 & 576 & 6.22 \\
\hline
\end{tabular}


(2002) and Michaels and Schilder's (1991) studies, in the prone posture, the up-right/down-left advantage was evident with the left hand $(50 \mathrm{msec})[F(1,11)=11.60, p=$ $.0059]$, but not with the right hand $(8 \mathrm{msec})[F(1,11)<$ $1.0]$. In the supine posture, this relation reversed, with the up-right/down-left advantage evident with the right hand $(40 \mathrm{msec})[F(1,11)=7.14, p=.0217]$, but not the left hand $(-16 \mathrm{msec})[F(1,11)<1.0]$. This hand posture effect did not interact with stimulus-set location $(F<1.0)$.

For the RT data, response hand interacted with response $\left[F(1,44)=8.26, M S_{\mathrm{e}}=3,450, p=.0062\right]$, and this interaction was qualified by a three-way interaction with hand posture $\left[F(1,44)=8.18, M S_{\mathrm{e}}=3,450, p=.0064\right]$. When subjects responded with the left hand, right responses ( $M \mathrm{~s}=556$ and $497 \mathrm{msec}$ for the prone and supine postures, respectively) were faster than left responses ( $M \mathrm{~s}=$ 564 and $515 \mathrm{msec}$, respectively) in both hand postures, whereas when subjects responded with the right hand, right responses $(M=520 \mathrm{msec})$ were faster than left responses $(M=528 \mathrm{msec})$ in the prone posture, but left responses $(M=531 \mathrm{msec})$ were faster than right responses $(M=$ $553 \mathrm{msec}$ ) in the supine posture.

For the PE data, hand posture showed a main effect, with responses more accurate in the supine posture $(3.03 \%)$ than in the prone posture $(4.81 \%)\left[F(1,44)=4.87, M S_{\mathrm{e}}=\right.$ $171.72, p=.0326]$, and it interacted with response $\left[F(1,44)=5.29, M S_{\mathrm{e}}=15.88, p=.0262\right]$. In the prone posture, PE was lower for right responses $(4.59 \%)$ than for left responses $(5.02 \%)$. However, in the supine posture, PE was lower for left responses $(2.73 \%)$ than for right responses (3.34\%). No interaction of mapping with hand posture and hand occurred $\left[F(1,44)=1.61, M S_{\mathrm{e}}=\right.$ $76.29, p=.2114]$.

Response eccentricity effect. The response eccentricity effect occurred, as indicated by a significant interaction of mapping and response-set location for both the RT and PE data $\left[F \mathrm{~s}(2,88)=27.42\right.$ and $15.02, M S_{\mathrm{e}} \mathrm{s}=$ 5,126 and 35.96, $p \mathrm{~s}=.0001$; see Figure 2]. An up-right/ down-left advantage of $35 \mathrm{msec}$ and $2.27 \%$ was found at the center location $[F \mathrm{~s}(1,44)=10.12$ and $14.78, p \mathrm{~s}=$ .0027 and .0004]. It increased to $50 \mathrm{msec}$ and $2.69 \%$ at the right location $[F \mathrm{~s}(1,44)=20.20$ and $15.16, p \mathrm{~s}<$ $.0003]$ and reversed to an up-left/down-right advantage of $22 \mathrm{msec}$ and $1.62 \%$ at the left location $[F \mathrm{~s}(1,44)=$ 9.22 and 3.63, $p \mathrm{~s}=.0040$ and .0634$]$. For RT, the response eccentricity effect did not interact with any other factors or combination of them $[F \mathrm{~s}(2,56)<1.19, p \mathrm{~s}>.3121]$, including the combination of response hand and hand posture $\left[F(2,56)=1.18, M S_{\mathrm{e}}=5,126, p=.3120\right]$. That is, the response eccentricity and hand posture effects on orthogonal SRC were additive for RT, as in Cho and Proctor's (2002) Experiment 1.

For PE, response-set location interacted significantly with response $\left[F(2,88)=3.83, M S_{\mathrm{e}}=15.04, p=.0254\right]$. The left responses $(3.40 \%$ and $3.97 \%$ for the right and center locations, respectively) were more accurate than the right responses ( $4.25 \%$ and $4.08 \%$, respectively) at the right and center locations, but the right responses $(3.56 \%)$ were more accurate than the left responses (4.25\%) at the left location. Response-set location and response hand interacted as well $\left[F(2,88)=3.38, M S_{\mathrm{e}}=19.78, p=\right.$ $.0386]$. For the left hand, PE was lower at the right location $(2.78 \%)$ than at the other locations $(3.40 \%$ and $3.79 \%$ at the center and left locations, respectively); for the right hand, PE was lower at the left location (4.12\%) than at the other locations $(4.65 \%$ and $4.87 \%$ at the center and right locations, respectively).

The five-way interaction of mapping, stimulus-set location, response-set location, response hand, and hand posture was significant for PE $\left[F(2,88)=3.70, M S_{\mathrm{e}}=\right.$ $19.52, p=.0287]$. When subjects responded with the right hand in the prone posture, the response eccentricity effect (i.e., the difference in the mapping effect between the left and right response-set locations) was larger with the upper stimulus set (11.68\%) than with the lower stimulus set $(3.96 \%)$. But in the supine posture, it was larger with the lower stimulus set (3.82\%) than with the upper stimulus set $(2.86 \%)$. When subjects responded with the left hand, the response eccentricity effect did not differ with the combination of the stimulus-set location and hand posture $(3.15 \%, 3.21 \%, 3.62 \%$, and $3.65 \%$ for the upper and lower stimulus sets in the prone posture and those in the supine posture, respectively).

Simon-type effect for stimulus-set location. The main effect of stimulus-set location for RT $[F(1,44)=$ $\left.6.56, M S_{\mathrm{e}}=1,264, p=.0139\right]$ indicated that $\mathrm{RT}$ was shorter when the stimulus set appeared on the upper part of the screen $(M=530 \mathrm{msec})$ than on the lower part $(M=535 \mathrm{msec})$. The interaction of stimulus-set location and response was not significant $[F(1,44)<1.0]$, indicating that there was no overall Simon-type effect for the irrelevant stimulus-set location. However, stimulusset location and response entered into higher order interactions with response-set location $[F(2,88)=8.43$, $M S_{\mathrm{e}}=867, p=.0004$; see Figure 3], and response hand and hand posture $\left[F(1,44)=10.59, M S_{\mathrm{e}}=1,523, p=\right.$ .0022 ; see Figure 4]. These interactions reveal Simontype effects that were modulated by response location (i.e., a response eccentricity effect) and hand/hand posture (i.e., a hand posture effect).

To examine these effects in more detail, stimulus-set location and response were collapsed into a variable of relation (upper-right/lower-left, upper-left/lower-right), and follow-up ANOVAs were conducted. The pattern of the response eccentricity effect for irrelevant stimulusset location was as follows: When subjects responded at the center location, there was no significant difference in mean RT for the upper-left/lower-right and upper-right / lower-left relations $[F(1,44)<1.0]$. But when subjects responded at the left location, RT was $8 \mathrm{msec}$ shorter for the upper-left/lower-right relation than for the upperright/lower-left relation $\left[F(1,44)=6.26, M S_{\mathrm{e}}=854\right.$, $p=.0161]$, and when subjects responded at the right response location, RT was $9 \mathrm{msec}$ longer for the upperleft/lower-right relation than for the upper-right/lowerleft relation $\left[F(1,44)=10.17, M S_{\mathrm{e}}=879, p=.0026\right]$. 

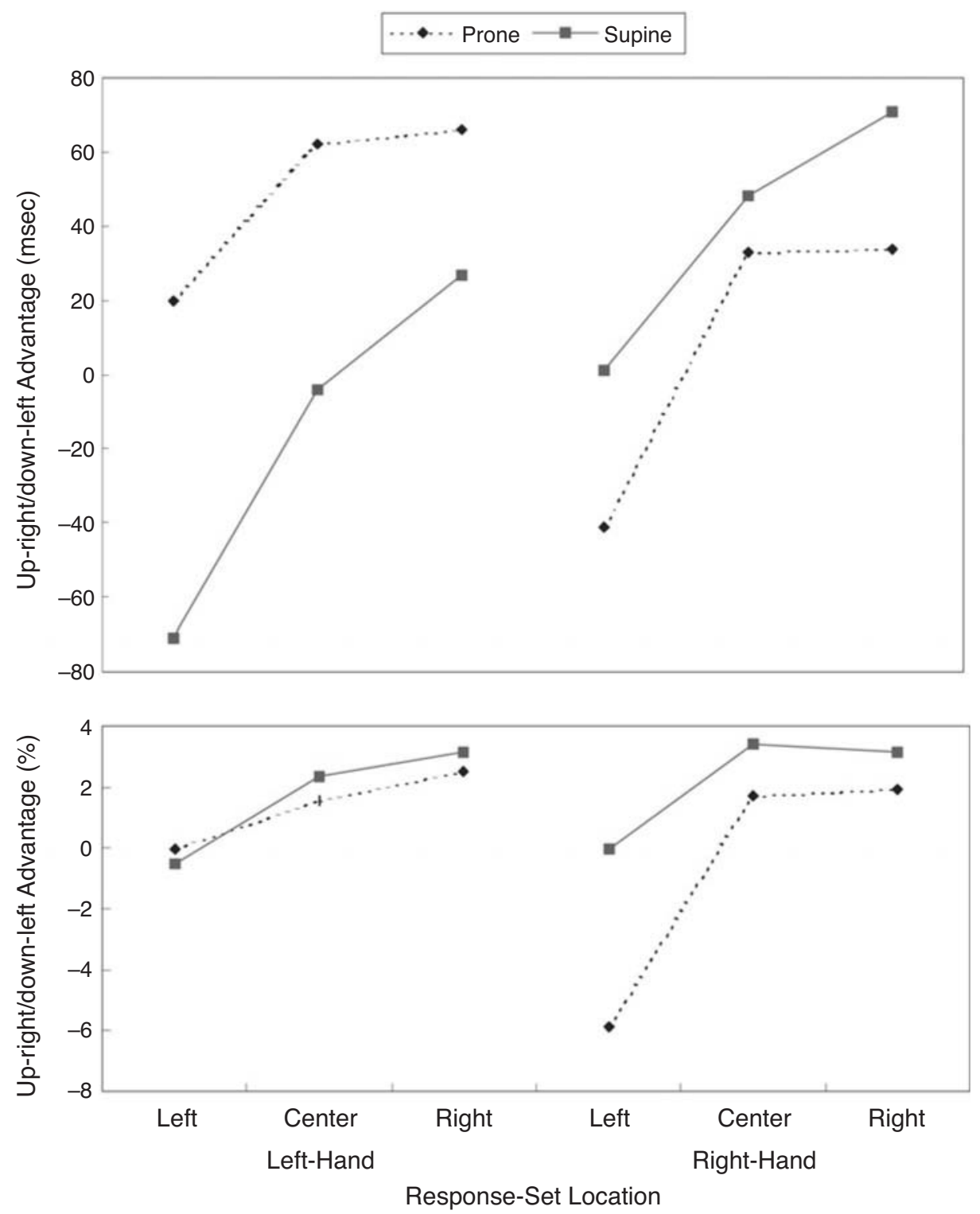

Figure 2. The up-right/down-left advantage as a function of response-set location and hand posture for mean reaction time and percent error in Experiment 1.

In other words, the irrelevant stimulus-set location variable produced a response eccentricity effect that was qualitatively similar to that for the relevant stimulus location variable, only smaller in magnitude.

The pattern of the hand posture effect was as follows: For the left hand, the upper-right/lower-left advantage was larger in the prone posture $(8 \mathrm{msec})$ than in the supine posture $(-10 \mathrm{msec})\left[F(1,22)=7.62, M S_{\mathrm{e}}=1,682\right.$, $p=.0146]$, although only the latter differed significantly from $0[F \mathrm{~s}(1,11)=2.09$ and $6.35, p \mathrm{~s}=.1759$ and .0285 , respectively]. In contrast, with the right hand, the upperright/lower-left advantage tended to be larger in the supine posture $(7 \mathrm{msec})$ than in the prone posture $(-4 \mathrm{msec})$
$\left[F(1,22)=3.69, M S_{\mathrm{e}}=1,364, p=.0678\right]$, although only the former differed significantly from $0\left[F_{\mathrm{S}}(1,11)=\right.$ 5.12 and $0.78, p \mathrm{~s}=.0448$ and .3966 , respectively]. This pattern is similar to the hand posture effect obtained for the relevant stimulus location variable, again with the differences being smaller. It is important to note that in the overall ANOVA, the significant four-way interaction of stimulus-set location, response, hand, and hand posture was not qualified by a higher order interaction with response-set location $[F(2,88)<1.0]$. Thus, the response eccentricity and hand posture effects for the irrelevant orthogonal Simon-type effect were approximately additive, as were those effects for the relevant stimulus loca- 


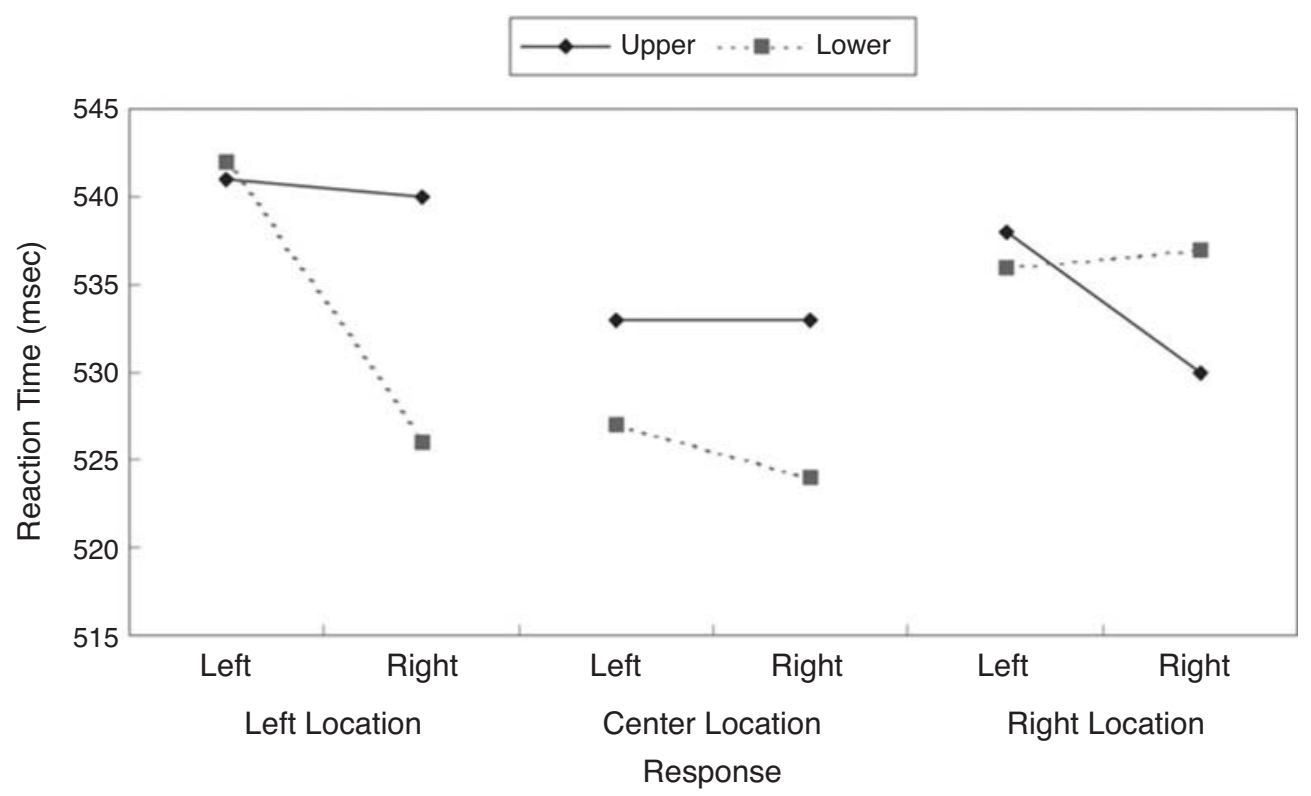

Figure 3. Mean reaction time as a function of stimulus-set location, response-set location, and response in Experiment 1.

tion variable. These results indicate that the upper-lower location of the stimulus set was coded asymmetrically, activating the response codes of corresponding polarity with respect to the position at which the responses were made and the body of the hand. These spatial codes affected performance in a qualitatively similar manner to those produced for the relevant stimulus-location variable.

\section{Discussion}

In agreement with prior studies, an overall advantage of $20 \mathrm{msec}$ for the up-right/down-left mapping was evident. As in Cho and Proctor's (2002) Experiment 1, this orthogonal SRC effect for RT was influenced independently by response eccentricity and hand posture. The up-right/down-left advantage was larger when subjects responded in the right hemispace than at midline, and it reversed to an up-left/down-right advantage in the left hemispace. This response eccentricity effect occurred regardless of whether the left or right hand was used or the hand was in a prone or supine posture. The orthogonal SRC effect was also influenced by the combination of responding hand and hand posture, with this hand posture effect occurring at all switch locations. These additive effects of response eccentricity and hand posture indicate that, as the multiple asymmetric codes account suggests, response location is coded in terms of multiple reference frames. Spatial codes for the response-set location were formed with respect to responding hand and the hemispace in which the responses were made, respectively, and influenced the polarity of the response codes.

Orthogonal Simon-type effects occurred between the stimulus-set location and response. That is, response ec- centricity and hand posture effects similar to those obtained for the relevant stimulus dimension were evident for the irrelevant upper and lower locations of the stimulus set. These orthogonal Simon-type effects show unambiguously that stimulus-set location was coded. The code for stimulus-set location could be formed as a consequence of its location relative to the centered fixation point (e.g., Hommel, 1993) or of a shift of attention, and probably also of the eyes, from that point to the fixation row (e.g., Umiltà \& Nicoletti, 1992). More generally, the Simon-type effects for stimulus-set location indicate that an irrelevant stimulus code can automatically activate a response code that corresponds to it only in polarity.

Despite unambiguous evidence that stimulus-set location was indeed coded, neither the overall orthogonal SRC effect for the relevant stimulus-location variable nor the response eccentricity and hand posture effects were influenced by stimulus-set location. These results indicate that stimulus-set location had no effect on the polarity of the stimulus codes. Despite the independence of the orthogonal SRC and Simon-type effects, the patterns of the response eccentricity and hand posture effects for both were qualitatively similar. Thus, stimulusset location and the location of the imperative stimulus influenced the response-selection process in an independent but similar manner. This result also indicates that, like the multiple spatial codes for response location, multiple spatial codes for stimulus location are formed relative to available reference frames and affect response selection additively (e.g., Cho \& Proctor, 2002; Lamberts et al., 1992; Proctor \& Pick, 1999).

But there is a difference in how the stimulus- and response-set codes influence the response-selection pro- 


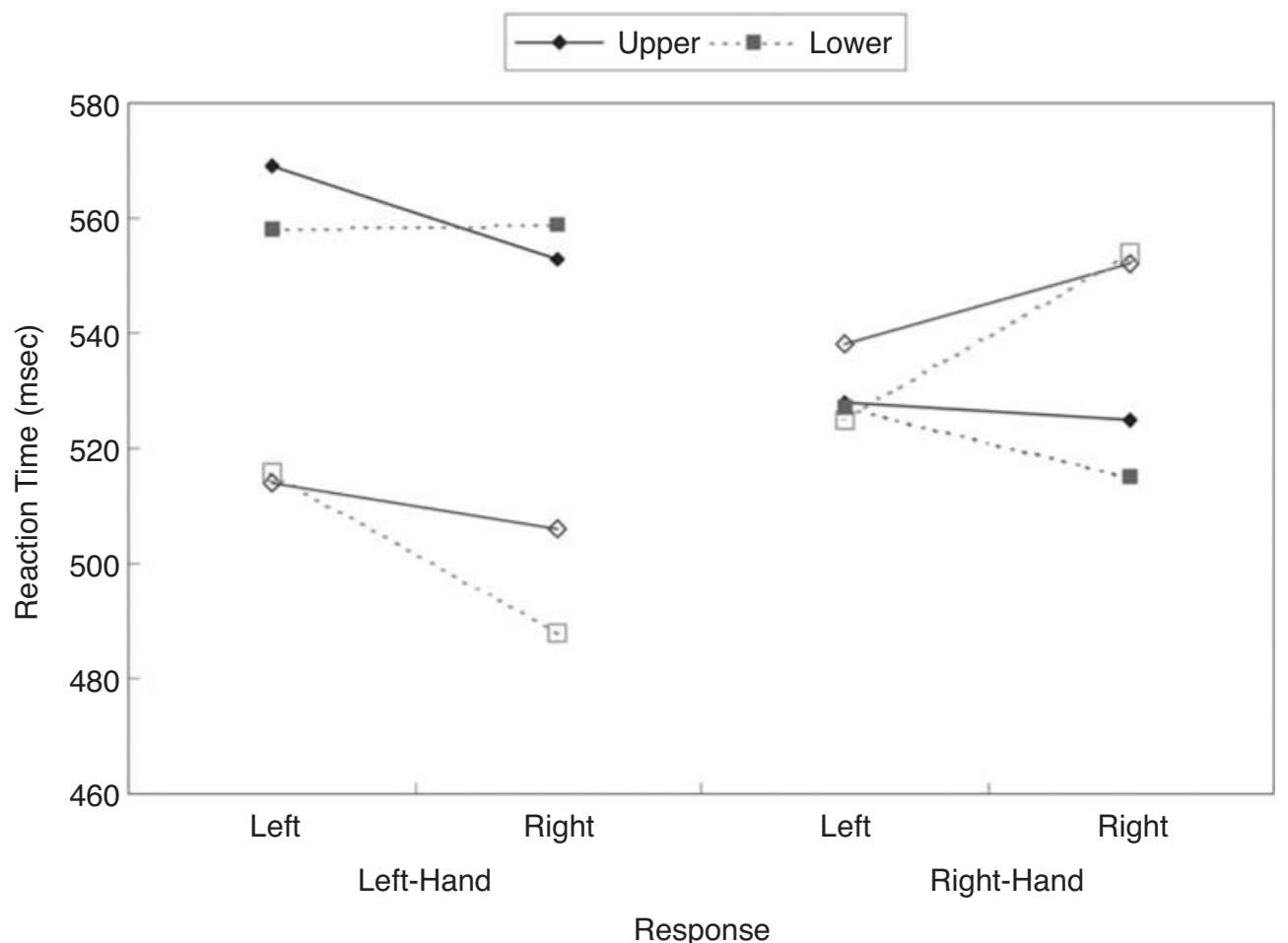

Figure 4. Mean reaction time as a function of stimulus-set location, response hand, and response for the prone posture (filled symbols) and supine posture (unfilled symbols) in Experiment 1.

cess. Stimulus-set location seems to be coded automatically, as for the Simon effect in general (see, e.g., Lu \& Proctor, 1995), and this code activates the response code of corresponding polarity, thus affecting performance. However, it does not affect the polarities of the spatial codes for the imperative stimuli, which implies that stimulus-set location is not represented as part of the task set. On the contrary, the spatial codes for responseset location do influence the polarities of the response codes and are part of the task set. There are at least two possible reasons for this difference. One is that stimulusset location was varied randomly from trial to trial, rather than blocked as response-set location was, which means that stimulus-set location was not known prior to a given trial. A more likely reason why stimulus-set location did not influence the polarity of stimulus codes is that, more generally, it is a task-irrelevant property and, as such, is not processed in the stage that determines the polarity of the stimulus codes (Cho \& Proctor, in press-b).

\section{EXPERIMENT 2}

Cho and Proctor (in press-a) found an influence of stimulus-set location on orthogonal SRC when it varied along the same dimension as the responses (i.e., up-down stimuli were mapped to left-right responses, and the stimulus set appeared in the left or right half of the screen). In their Experiment 1, the up-right/down-left advantage was
$21 \mathrm{msec}$ when the stimulus set was on the left side of the screen (and the centered response set was to the right of the stimulus set) but only $8 \mathrm{msec}$ when it was on the right side (and the centered response set was to the left of the stimulus set). This pattern of results is similar to that found when the active switch is located to the left or right of an inactive switch (Proctor \& Cho, 2003; Weeks et al., 1995), suggesting that it is a consequence of coding the location of the response set relative to the stimulus set.

For the response eccentricity effect, response location relative to body midline is confounded with response location relative to the stimulus display because the display and subject remain at constant positions, and only the position at which the responses are made is varied. Cho and Proctor's (in press-a) Experiment 3 separated the effects of these two variables by covarying, along with the location of the response set, either the position of the display screen (the midline-referent condition; e.g., for the right response set, the display screen was shifted to align with that position) or that of the subject (the display-referent condition; e.g., for the right response set, the subject's body was aligned with the response position, and the display screen was to the left of the subject). In the display-referent condition, the response eccentricity effect occurred regardless of response mode (i.e., for left- and right-hand unimanual switch movements and bimanual keypresses), but in the midlinereferent condition, the response eccentricity effect was 
not significant. This pattern of results suggests that the response eccentricity effect is due primarily to location of the response set relative to the display.

However, for unimanual responses made with the left or right hand, a nonsignificant tendency suggested that the response location relative to body midline influenced the orthogonal SRC effect. The present Experiment 2 provided a stronger test of whether coding relative to body midline also affects orthogonal SRC by using a factorial design for which responses were made in the left or right hemispace and the stimulus display was aligned with the response/hand position or shifted to the left or right of it. The key questions were whether (1) a significant effect of response location relative to midline would be evident in addition to the effect of response location relative to the display, (2) these two effects would be additive, as the multiple asymmetric codes account predicts, and (3) the effect of response location relative to body midline would be found for bimanual keypresses as well as for unimanual switch movements.

\section{Method}

Subjects. Forty-eight new students, from the same pool as Experiment 1 and satisfying the same restrictions, participated. The subjects were randomly assigned to the three different response mode groups: left and right unimanual switch movement, and bimanual keypress, and within those groups, the hand position was left for half of the subjects and right for the other half.

Apparatus and Stimuli. The apparatus and stimuli were identical to Experiment 1, except that the fixation row " +++ " always appeared at the center of the screen without the onset of the fixation point "**." For the bimanual response mode, the leftmost and rightmost response buttons on the MEL response box were used, with subjects pressing the left button with the left index finger and the right button with the right index finger. For the unimanual responses, as in Experiment 1, a toggle switch was pushed left or right with the left hand (the left-hand unimanual response mode) or the right hand (the right-hand unimanual response mode). For half of the subjects in each response-mode group, the response apparatus was placed $20 \mathrm{~cm}$ to the left of body midline, and for the other half, it was placed $20 \mathrm{~cm}$ to the right of body midline.

Procedure. Subjects were asked to align their body midline with an arrow mark depicted on the table. The display location was varied relative to the position of the response apparatus, which was either in the left hemispace or right hemispace for all conditions experienced by a subject. The computer monitor was placed at three different locations: center, at which the monitor was in line with the response apparatus, or $20 \mathrm{~cm}$ to the left or right of the response apparatus. When the monitor was placed to either side of body midline (the left and center display locations for subjects responding in the left hemispace, and the right and center display locations for subjects responding in the right hemispace), the subjects were allowed to move their heads to see it, but not to turn their bodies toward it.

The experiment consisted of two three-block sessions with a 2min rest period between them. Half of the subjects in each group performed the first session with the up-left/down-right mapping and the second session with the up-right/down-left mapping. The other half performed in the opposite order. Half of the subjects began with the computer monitor placed to the right of the response apparatus and progressed to the left, whereas the other half began with the computer monitor placed to the left of the response apparatus and progressed to the right. Each subject performed 10 practice trials when the new or different mapping was introduced, and each block consisted of 50 trials. After each block was completed, a 30 -sec rest period was given. During the rest period, the experimenter moved the monitor to the appropriate location for the next block.

At the beginning of each trial, the fixation row was presented in the center of the screen for $500 \mathrm{msec}$. The imperative stimulus was presented above or below the fixation point, both of which remained on until the subject responded. An incorrect response was followed by a 500 -msec feedback tone. The fixation point for the next trial came on $1 \mathrm{sec}$ after the response when it was correct and after the feedback tone when the response was incorrect.

\section{Results}

With the same exclusion criteria as in Experiment 1, $0.47 \%$ of the trials were removed from analysis. Mean RT and PE were calculated for each subject as a function of mapping (up-right/down-left, up-left/down-right), response (left, right), and display location (left, center, right). ANOVAs were conducted on the mean RT and PE data, with those variables as within-subjects factors and response mode (left, right, bimanual) and hand position (left, right) as between-subjects factors (see Table 2).

Overall mapping effect. As in Experiment 1, there was an overall up-right/down-left advantage: RT was shorter and PE less for the up-right/down-left mapping $(M \mathrm{~s}=361 \mathrm{msec}$ and $1.75 \%)$ than for the up-left/downright mapping $(M \mathrm{~s}=379 \mathrm{msec}$ and $2.58 \%)[F \mathrm{~s}(1,42)=$ 11.53 and $6.09, M S_{\mathrm{e}} \mathrm{s}=3,997$ and 16.14 , and $p \mathrm{~s}=.0015$ and .0178]. Mapping interacted with response for both measures $\left[F \mathrm{~s}(1,42)=6.97\right.$ and $6.24, M S_{\mathrm{e}} \mathrm{s}=1,285$ and $11.42, p \mathrm{~s}=.0116$ and .0165$]$. The up-right/down-left advantage was smaller in RT but larger in PE for right responses $(10 \mathrm{msec}$ and $1.52 \%)$ than for left responses (26 msec and $0.12 \%$ ). This tendency for RT and PE to

Table 2

Mean Reaction Times (RTs, in Milliseconds) and

Percentages of Error (PEs) in Experiment 2 as a Function of Mapping, Display Location (Left, Center, Right), Response Hand, and Hand Position

\begin{tabular}{|c|c|c|c|c|c|c|}
\hline \multirow[b]{2}{*}{ Hand and Mapping } & \multicolumn{2}{|c|}{ Left } & \multicolumn{2}{|c|}{ Center } & \multicolumn{2}{|c|}{ Right } \\
\hline & RT & $\overline{\mathrm{PE}}$ & RT & $\mathrm{PE}$ & RT & $\mathrm{PE}$ \\
\hline
\end{tabular}

Left Position

Left Hand

Up-right/down-left

Up-left/down-right

$\begin{array}{llllll}334 & 1.50 & 341 & 1.00 & 365 & 3.50 \\ 350 & 2.25 & 335 & 0.75 & 333 & 2.25 \\ & & & & & \\ 382 & 3.31 & 407 & 2.00 & 440 & 3.25 \\ 414 & 0.76 & 418 & 1.01 & 386 & 1.00 \\ & & & & & \\ 283 & 0.25 & 284 & 1.75 & 297 & 1.50 \\ 333 & 5.52 & 316 & 3.26 & 303 & 2.26\end{array}$

Up-right/down-left

Up-left/down-right

Bimanual

Up-right/down-left

Up-left/down-right

Right Position

Left Hand

$\begin{array}{lllllll}\text { Up-right/down-left } & 416 & 0.76 & 434 & 1.80 & 439 & 1.63\end{array}$

$\begin{array}{lllllll}\text { Up-left/down-right } & 495 & 3.88 & 480 & 3.77 & 438 & 2.54\end{array}$

Right Hand

$\begin{array}{lllllll}\text { Up-right/down-left } & 358 & 1.75 & 373 & 2.25 & 362 & 1.75\end{array}$

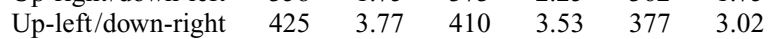

Bimanual

$\begin{array}{lllllll}\text { Up-right/down-left } & 315 & 0.50 & 321 & 1.26 & 335 & 1.75\end{array}$

$\begin{array}{lllllll}\text { Up-left/down-right } & 352 & 2.27 & 326 & 1.53 & 330 & 1.00\end{array}$



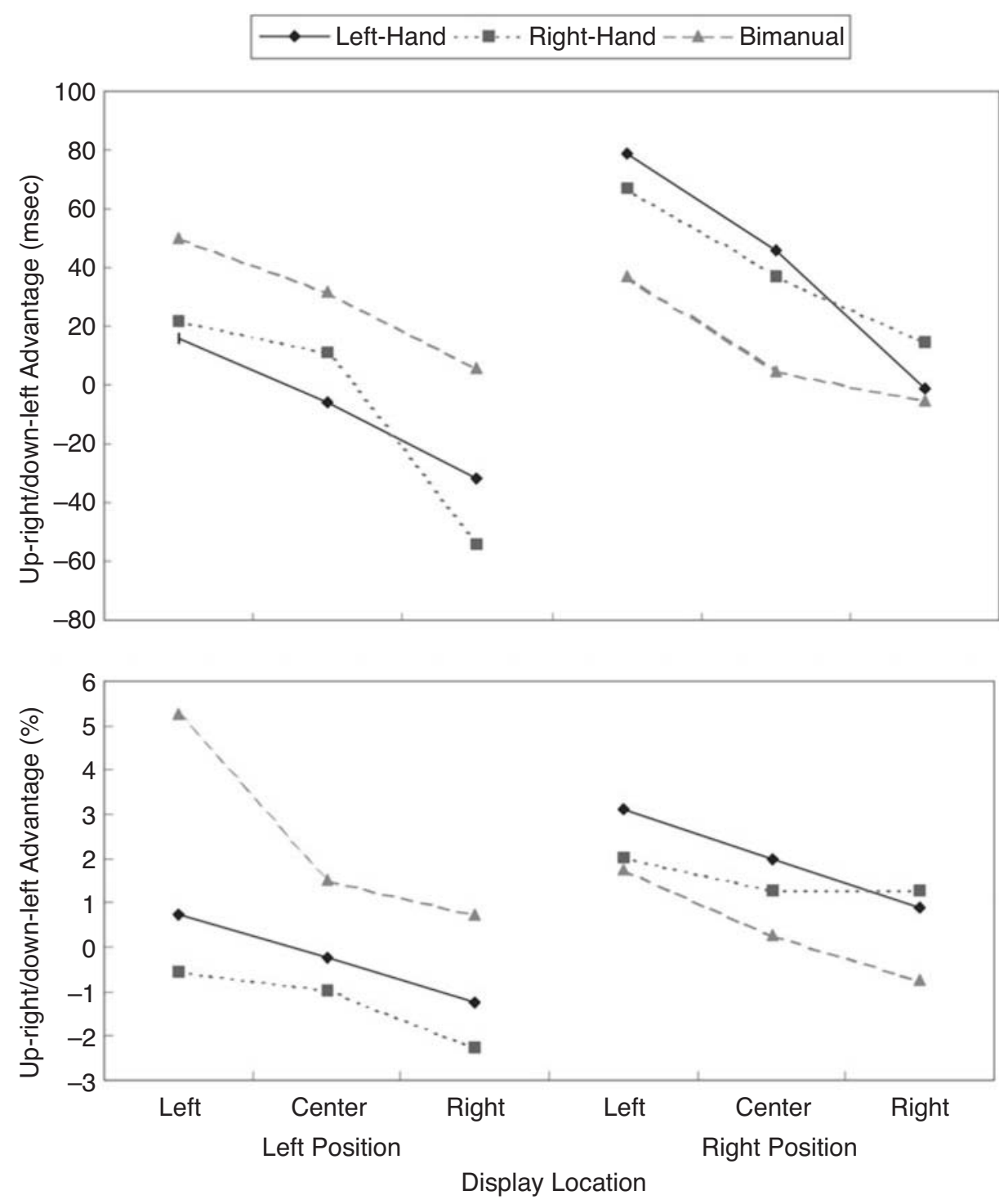

Figure 5. The up-right/down-left advantage as a function of display location relative to responseset, response hand, and hand position for reaction time and percent error in Experiment 2.

show opposing results is similar to patterns of results in Proctor and Cho (2003).

Stimulus-set location effect. As in Cho and Proctor's (in press-a) Experiment 3, the orthogonal SRC effect varied as a function of display location for both RT and PE (see Figure 5) $\left[F \mathrm{~s}(2,84)=30.05\right.$ and $10.09, M S_{\mathrm{e}} \mathrm{s}=$ $1,296$ and 6.32, $p \mathrm{~s}<.0001]$. An up-right/down-left advantage of $21 \mathrm{msec}$ and $0.63 \%[F \mathrm{~s}(1,42)=8.59$ and $1.66, p \mathrm{~s}=.0054$ and .2043$]$ occurred when the stimulus display was aligned with the response apparatus. This up-right/down-left advantage increased to $45 \mathrm{msec}$ and $2.06 \%$ when the display was to the left of the apparatus $[F \mathrm{~s}(1,42)=36.59$ and $19.38, p \mathrm{~s}<.0001]$, but reversed to an up-left/down-right advantage of $11 \mathrm{msec}$ and $0.22 \%$ when the display was to the right of the apparatus
$\left[F_{\mathrm{s}}(1,42)=4.84\right.$ and $0.29, p \mathrm{~s}=.0334$ and .5958$]$. This effect, which follows the pattern expected on the basis of coding the response location relative to the display, did not interact with response mode, hand position, or both variables together $\left(F_{\mathrm{s}} \leq 1.06\right)$.

Body midline effect. There was also an effect of response location relative to body midline, as indicated by a significant interaction of mapping with hand position in the RT data $\left[F(1,42)=6.16, M S_{\mathrm{e}}=3,997, p=\right.$ $.0172]$. The up-right/down-left advantage was a nonsignificant $5 \mathrm{msec}$ when subjects responded in the left hemispace $[F(1,21)<1.0]$, but a significant $31 \mathrm{msec}$ when they responded in the right hemispace $[F(1,21)=$ $18.25, p=.0003]$. The interaction of mapping and hand position with response mode was significant for both RT 
and PE $\left[F \mathrm{~s}(2,42)=4.13\right.$ and $5.30, M S_{\mathrm{e}} \mathrm{s}=3,997$ and $16.14, p \mathrm{~s}=.0230$ and .0088$]$. For the unimanual modes, the up-right/down-left advantage was larger in the right hemispace (40 msec and $1.76 \%$ ) than in the left hemispace $(-7 \mathrm{msec}$ and $-0.75 \%)$. However, for the bimanual mode, there was no significant difference in the upright/down-left advantage when subjects responded in the left and right hemispaces [ $29 \mathrm{msec}$ and $2.51 \%$, and $12 \mathrm{msec}$ and $0.43 \%$, respectively; $F \mathrm{~s}(1,14) \leq 1.99, p \mathrm{~s} \leq$ .1799].

Other significant effects. No other effects were significant for PE, but several were for RT. RT was shorter for the bimanual response mode $(M=316 \mathrm{msec})$ than for the right-hand $(M=397 \mathrm{msec})$ and left-hand $(M=$ $397 \mathrm{msec}$ ) unimanual response modes $[F(2,42)=12.64$, $\left.M S_{\mathrm{e}}=32,852, p<.0001\right]$, and RT was shorter when the response apparatus was positioned in the left hemispace $(M=351 \mathrm{msec})$ than in the right hemispace $(M=$ $388 \mathrm{msec})\left[F(1,42)=5.82, M S_{\mathrm{e}}=32,582, p=.0202\right]$. Hand position interacted with response mode $[F(1,42)=$ $\left.6.53, M S_{\mathrm{e}}=32,582, p=.0034\right]$. For the left-hand unimanual and bimanual keypress responses, RT was shorter at the left hemispace $(M \mathrm{~s}=343 \mathrm{msec}$ and $303 \mathrm{msec}$ for the left-hand unimanual and bimanual response modes, respectively) than in the right hemispace $(M \mathrm{~s}=450 \mathrm{msec}$ and $330 \mathrm{msec}$, respectively), whereas for the right-hand response mode, responses were faster in the right hemispace $(M=384 \mathrm{msec})$ than in the left hemispace $(M=$ $409 \mathrm{msec})$.

Display location interacted with response $[F(2,84)=$ $\left.4.99, M S_{\mathrm{e}}=245, p=.0089\right]$. In the right and center display locations, right responses $(M=364 \mathrm{msec})$ were faster than left responses $(M \mathrm{~s}=373 \mathrm{msec})$, whereas in the left display location, RT was identical for left and right responses $(M \mathrm{~s}=372 \mathrm{msec})$. Response mode also interacted with response $\left[F(2,42)=4.83, M S_{\mathrm{e}}=1,244, p=\right.$ $.0130]$. For the left-hand unimanual responses, left responses $(M=393 \mathrm{msec})$ were faster than right responses $(M=400 \mathrm{msec})$. For the right-hand unimanual and bimanual response modes, right responses $(M \mathrm{~s}=392$ and $308 \mathrm{msec}$, respectively) were faster than left responses ( $M \mathrm{~s}=401$ and $324 \mathrm{msec}$, respectively). The interaction of hand position and response was significant $[F(1,42)=$ 4.33, $\left.M S_{\mathrm{e}}=1,244, p=.0436\right]$. In the right hemispace, the mean RTs for the two responses were identical $(M=$ $388 \mathrm{msec})$, but in the left hemispace, right responses $(M=346 \mathrm{msec})$ were faster than left responses $(M=$ $358 \mathrm{msec})$.

\section{Discussion}

Although subjects responded at positions in the right or left hemispace, in contrast to the midline position used in Cho and Proctor's (in press-a) Experiment 3, orthogonal SRC varied systematically as a function of whether the display was aligned with the response position or placed to the left or right of it. The pattern of results, an increase in the up-right/down-left advantage when the display was to the left of the response position and a reversal to an up-left/down-right advantage when the display was to the right, implies that a spatial code for response-set location is formed with reference to the stimulus set and that orthogonal SRC is affected by the polarities of the response codes within this reference frame.

Hand position influenced the orthogonal SRC effect significantly in the two unimanual response modes. Regardless of the response hand, an up-left/down-right advantage of $7 \mathrm{msec}$ and $0.24 \%$ occurred in the left hemispace, whereas an up-right/down-left advantage of $40 \mathrm{msec}$ and $1.25 \%$ occurred in the right hemispace. This pattern is similar to the nonsignificant pattern obtained in Cho and Proctor's (in press-a) Experiment 3 (a 17-msec and 0.33\% up-right/down-left advantage in the left hemispace and a 40 -msec and $1.77 \%$ up-right/down-left advantage in the right hemispace), in which responses were made at body midline. The two experiments together, therefore, indicate that, for unimanual responses, the orthogonal SRC effect is moderated by whether responding is in the left or right hemispace, and the pattern suggests that the response position is coded relative to body midline as well as to the stimulus set. Moreover, the lack of an interaction of this effect with that due to display location implies that the polarities of the response codes relative to body midline affect performance independently from those for the response codes relative to the display, producing additive influences on orthogonal SRC.

For the bimanual response mode, also as in Cho and Proctor's (in press-a) Experiment 3, there was no significant effect of hand position on orthogonal SRC. The absence of a significant effect suggests that response location is not coded relative to body midline for keypress responses made with the left and right hands. However, this suggestion must be taken with caution because both the RT and PE data showed nonsignificant tendencies toward an effect of hand position that was opposite that of the effect for unimanual responses.

\section{GENERAL DISCUSSION}

Both Experiments 1 and 2 showed an overall up-right / down-left mapping advantage, in agreement with previous studies. Experiment 1 replicated the response eccentricity effect, a larger up-right/down-left advantage when subjects responded in the right hemispace than in the left hemispace. It also showed the standard hand posture effect, with the up-right/down-left advantage being larger for unimanual responses made with the left hand than with the right hand in the prone posture, but the opposite in the supine posture. As in earlier studies, the hand posture effect on RT did not interact with the response eccentricity effect. These results illustrate the robustness of the major findings that are obtained for orthogonal SRC effects.

\section{Primary Outcomes}

In Experiment 1, stimulus-set location did not interact with left-right response when subjects responded at mid- 
line, consistent with Cho and Proctor's (in press-b) study. Moreover, there was no overall interaction of stimulusset location and response when collapsed across all three response-set locations, indicating that there was no overall Simon-type effect for the irrelevant stimulus-location variable corresponding to the up-right/down-left advantage obtained for the relevant stimulus-location mapping. However, orthogonal Simon-type effects were evident in significant higher order interactions of stimulus-set location and response with response-set location and hand posture. These interactions showed response eccentricity and hand posture effects for the irrelevant stimulus-set location that were qualitatively similar to those obtained for the relevant stimulus-location mapping. This pattern of results indicates clearly that stimulus-set location was coded, and that the spatial codes for it were also asymmetric.

Even though stimulus-set location was coded and affected performance, it did not influence the orthogonal SRC effect for the relevant S-R mapping. Not only was the overall up-right/down-left advantage unaffected by stimulus-set location, as in Cho and Proctor's (in press-b) experiment that examined only body midline responses, but so were the response eccentricity and hand posture effects for the relevant $\mathrm{S}-\mathrm{R}$ mapping. That is, the pattern of orthogonal SRC effects as a function of response eccentricity and hand posture was similar when the stimulus set was in the upper half of the screen to when it was in the lower half. Thus, Experiment 1 establishes that the lack of influence of stimulus-set location on orthogonal SRC observed by Cho and Proctor (in press-b) was not a consequence of subjects failing to code stimulus-set location and that there is no indication of any influence of stimulus-set location on orthogonal SRC when the response eccentricity and hand posture effects are considered in addition to the overall up-right/down-left advantage.

When response eccentricity is manipulated, the location of the response set changes relative to both the display and body midline. To dissociate these two aspects of relative location, Cho and Proctor (in press-a) examined conditions in which either the location of the display or that of the subject remained aligned with the response apparatus at the respective eccentricities. That experiment showed a significant effect of response-set location relative to the display but only a nonsignificant trend for response-set location relative to body midline (and that only for unimanual responses). The complete factorial design used in Experiment 2 confirmed the importance of response-set location relative to the display. Although all responding in Experiment 2 was in the right or left hemispace, manipulation of stimulus-set location about the response-set location had strong, significant effects. The results also showed that the pattern suggestive of an effect of response-set location relative to body midline apparently is real: Hand position had a significant effect on orthogonal SRC, with the up-right/down-left advantage being larger when responding in the right hemispace than in the left hemispace. This effect of coding based on an egocentric reference frame did not interact with that based on coding of response location relative to the display. Moreover, it was evident only for unimanual switch movements, made with either the left or right hand, and not for bimanual keypresses, which showed nonsignificant tendencies in the opposite direction.

\section{Implications for the Multiple Asymmetric Codes Account}

As indicated in the introduction, the majority of prior evidence favors an account in terms of correspondence of asymmetric codes for both the overall up-right/downleft advantage and the modifications of this orthogonal SRC effect by other variables. With respect to the multiple asymmetric codes account, the present results imply the following.

First, stimulus-set location is coded, and these codes are asymmetric, as indicated by the orthogonal Simon-type effects that vary as a function of response eccentricity and hand posture. This finding is important because it indicates that spatial information is coded asymmetrically even when it is irrelevant (as defined by the task instructions) and activates the response codes of corresponding polarity. Thus, asymmetric coding, and a benefit of correspondence of asymmetric stimulus and response codes, is not restricted to relevant information.

Second, the stimulus-set location code has no influence on the asymmetric coding of the target location, as indicated by the lack of interaction of stimulus-set location with the overall up-right/down-left advantage, the response eccentricity effect, or the hand posture effect for the relevant $\mathrm{S}-\mathrm{R}$ mapping. The implication is that which of the two relevant stimulus locations is coded as positive and which as negative is uninfluenced by other visual information available in the display. The study by Cho and Proctor (in press-b) ruled out the possibility that the lack of effect of stimulus-set location on orthogonal SRC for the relevant dimension was due to a property of the vertical dimension, and Experiment 1 shows that it is not due to a failure to code stimulus-set location. Rather, the asymmetric coding of stimulus location seems to be generally impervious to the visual context.

Third, response-set location relative to the display causes, at least in part, the asymmetric response codes that produce the response eccentricity effect on orthogonal SRC. For both unimanual switch movements and left-right keypresses, the orthogonal SRC effect is affected by display location in a manner consistent with this proposition. The effect size for keypresses $(49 \mathrm{msec})$ is the same as the size of the response eccentricity effect obtained by Proctor and Cho (2003), suggesting that the response eccentricity effect for keypress responses is due entirely to coding response location relative to the display. For unimanual switch movements, the orthogonal SRC effect is influenced as well by response-set location relative to body midline. The size of the response eccentricity effect for unimanual switch movements reported across different studies ranges from $49 \mathrm{msec}$ (Weeks et al., 1995) to $91 \mathrm{msec}$ (Cho \& Proctor, 2002), in agree- 
ment with the present findings suggesting that, in this case, codes formed with respect to both the display and body midline affect performance.

Fourth, the contributions from each reference frame combine additively. This was evident for RT in Experiment 1 for the up-right/down-left advantage, response eccentricity, and hand posture effects for the relevant stimulus location, and the Simon-type response eccentricity and hand posture effects for the irrelevant stimulusset location, none of which interacted with each other. It was also evident in Experiment 2, where the effect of coding relative to body midline for unimanual responses did not interact with the effect of coding relative to the display location. In general, the correspondences of asymmetric codes relative to each reference frame contribute independently to performance, with the direction and magnitude of effects determined by the relative frequency of codes of each polarity.

Three differences in results across conditions raise questions to which we offer tentative answers. The first is why stimulus-set location did not show an overall upper-right/lower-left Simon-type effect similar to the up-right/down-left advantage obtained for the relevant S-R mapping, although it did show response eccentricity and hand posture Simon-type effects. This finding can be explained in terms of dual-route models of SRC and Simon effects, which are currently the most widely advocated (e.g., Hommel \& Prinz, 1997). One route is that of intentional response selection, also called the translational or conditional route, which is based on short-term links between stimuli and responses, or general response-selection rules, which are defined for the specific task (e.g., Zorzi \& Umiltà, 1995). The other is an automatic activation route, also called the direct or unconditional route, by way of which a stimulus activates its corresponding response through long-term $\mathrm{S}-\mathrm{R}$ links acquired from previous experience. Within the context of dual-route models, the present results suggest that the up-right/down-left advantage, which is found only for the relevant $\mathrm{S}-\mathrm{R}$ mapping, is due to the short-term, task-defined links of the intentional route. The response eccentricity and hand posture effects found for the irrelevant relation between stimulus-set location and response are due to the long-term links of the automatic route. The larger response eccentricity and hand posture effects on orthogonal SRC for the relevant S-R mapping may reflect a contribution from the short-term links in addition to that from the long-term links.

The second question is why stimulus-set location does not affect the asymmetric coding of the alternative stimuli similar to how response-set location affects the asymmetric coding of the alternative responses. That is, why is the up-right/down-left advantage for the relevant $\mathrm{S}-\mathrm{R}$ mapping no larger when the stimulus set is in the upper position on the display screen than when it is in the lower position? This outcome can also be explained in terms of dual-route models: As described above, stimulus-set location exerts an effect on response selection by way of the direct, or automatic, activation route, but this factor is not part of the task-set representation that yields the coding of the relevant stimulus-location information. More generally, there is no reason why stimulus-set location should influence the polarities of the relevant stimulus codes. In contrast, to select and execute a correct response efficiently, the response situation (such as the previous responses, the response location, and the relation between the hand and response switch) must be taken into consideration (Hommel, 1997). Accordingly, consideration of the response situation sets the state of the cognitive processor, including the polarity of the response codes. Thus, response-set location will be coded as part of the task and will influence both intentional translation and direct activation, although stimulus-set location will not.

The third question is why performance was affected significantly by coding response location relative to body midline when the responses were unimanual switch movements, but not when they were keypresses made with the left and right hands. As mentioned in the preceding paragraph, the subject must take into consideration the entire response situation, including the response location, in order to respond efficiently. Different response modes require that different states of the cognitive processor be set up, and the same kinds of information are not necessarily involved in the response-selection process for all response modes (see Hommel, 1997). If consideration of response location relative to body midline is meaningful in a particular situation for setting the state of the cognitive processor, location relative to body midline will be represented and will influence the polarities of the response codes. Otherwise, it will not be represented and will have no influence. Perhaps response location relative to body midline is coded for unimanual switch movements because both responses require actions at the single location where the switch is grasped. For keypresses made with the left and right hands, however, the effectors are located at different positions within the hemispace, which makes coding relative to body midline less important.

\section{Conclusion}

This study demonstrates that the orthogonal SRC effect is due to general properties of spatial coding. The orthogonal SRC effect varied systematically with response location relative to multiple reference frames, even without changing motoric properties of the response hand(s). The multiple spatial codes for response location relative to stimulus-set location, response hand, and body midline increased the polarities of their corresponding response codes independently, affecting the orthogonal SRC effect in an additive manner. However, the spatial codes for response location were not necessarily formed relative to every possible reference frame. Different kinds of location information seem to be required to set up the states of the response-selection process for different response modes.

Also, as in SRC and Simon effects for parallel S-R dimensions, multiple spatial codes for stimulus location 
were formed relative to available frames of reference, and these spatial codes influenced the response-selection process independently. However, unlike the polarities of the response codes, the polarities of the stimulus codes were unaffected by stimulus-set location. In sum, the obtained results are consistent with the assumption of the multiple asymmetric codes account that multiple spatial codes for stimulus and response locations are formed relative to available reference frames and influence the response-selection process independently.

\section{REFERENCES}

Adam, J. J., Boon, B., Paas, F. G. W. C., \& Umiltà, C. (1998). The upright/down-left advantage for vertically oriented stimuli and horizontally oriented responses: A dual-strategy hypothesis. Journal of Experimental Psychology: Human Perception \& Performance, 24, 1582-1595.

BAUER, D. W., \& Miller, J. (1982). Stimulus-response compatibility and the motor system. Quarterly Journal of Experimental Psychology, 34A, 367-380.

Chase, W. G., \& Clark, H. H. (1971). Semantics in the perception of verticality. British Journal of Psychology, 62, 311-326.

Cho, Y. S., \& Proctor, R. W. (2002). Influences of hand posture and hand position on compatibility effects for up-down stimuli mapped to left-right responses: Evidence for a hand referent hypothesis. Perception \& Psychophysics, 64, 1301-1315.

CHO, Y. S., \& Proctor, R. W. (2003). Stimulus and response representations underlying orthogonal stimulus-response compatibility effects. Psychonomic Bulletin \& Review, 10, 45-73.

CHo, Y. S., \& Proctor, R. W. (in press-a). Representing response position relative to display location: Influence on orthogonal stimulusresponse compatibility. Quarterly Journal of Experimental Psychology.

Cho, Y. S., \& Proctor, R. W. (in press-b). Stimulus-set location does not affect orthogonal stimulus-response compatibility. Psychological Research/Psychologische Forschung.

Clark, H. H. (1973). Space, time, semantics and the child. In T. E. Moore (Ed.), Cognitive development and the acquisition of language (pp. 27-63). New York: Academic Press.

Hommel, B. (1993). The role of attention for the Simon effect. Psychological Research/Psychologische Forschung, 55, 208-222.

Hommel, B. (1997). Toward an action-concept model of stimulusresponse compatibility. In B. Hommel \& W. Prinz (Eds.), Theoretical issues in stimulus-response compatibility (pp. 281-320). Amsterdam: Elsevier, North-Holland.

Hommel, B., \& LiPPA, Y. (1995). S-R compatibility due to contextdependent spatial stimulus coding. Psychonomic Bulletin \& Review, 2, 370-374.

HOMMEL, B., \& Prinz W. (EDs.) (1997). Theoretical issues in stimulusresponse compatibility. Amsterdam: Elsevier, North-Holland.

Just, M. A., \& CARPENTER, P. A. (1975). The semantics of locative information in pictures and mental images. British Journal of Psychology, 66, 427-441.
Lamberts, K., Tavernier, G., \& D'Ydewalle, G. (1992). Effects of multiple reference points in spatial stimulus-response compatibility. Acta Psychologica, 79, 115-130.

LIPPA, Y., \& ADAM, J. J. (2001). An explanation of orthogonal S-R compatibility effects that vary with hand or response position: The end-state comfort hypothesis. Perception \& Psychophysics, 63, 156174.

Lu, C.-H., \& Proctor, R. W. (1995). The influence of irrelevant location information on performance: A review of the Simon and spatial Stroop effects. Psychonomic Bulletin \& Review, 2, 174-207.

Michaels, C. F. (1989). S-R compatibilities depend on eccentricity of responding hand. Quarterly Journal of Experimental Psychology, 41A, 262-272.

Michaels, C. F., \& Schilder, S. (1991). Stimulus-response compatibilities between vertically oriented stimuli and horizontally oriented responses: The effects of hand position and posture. Perception \& Psychophysics, 49, 342-348.

PROCTOR, R. W., \& CHO, Y. S. (2003). Effects of relative position and response eccentricity on orthogonal stimulus-response compatibility with joystick and keypress responses. Quarterly Journal of Experimental Psychology, 56A, 309-327.

PROCTOR, R. W., \& PICK, D. F. (1999). Deconstructing Marilyn: Robust effects of face contexts on stimulus-response compatibility. Memory \& Cognition, 27, 986-995.

Proctor, R. W., \& REEVE, T. G. (EDS.) (1990). Stimulus-response compatibility: An integrated perspective. Amsterdam: Elsevier, NorthHolland.

Proctor, R. W., Reeve, T. G., \& Van Zandt, T. (1992). Salient-features coding in response selection. In G. E. Stelmach \& J. Requin (Eds.), Tutorials in motor behavior 2 (pp. 727-741). Amsterdam: Elsevier, North-Holland.

Roswarski, T. E., \& Proctor, R. W. (1996). Multiple spatial codes and temporal overlap in choice-reaction tasks. Psychological Research, 59, 196-211.

SEYMOUR, P. H. K. (1974). Asymmetries in judgments of verticality. Journal of Experimental Psychology, 102, 447-455.

UMiltà, C., \& NicOLETTI, R. (1992). An integrated model of the Simon effect. In J. Alegria, D. Holender, J. Junça de Morais, \& M. Radeau (Eds.), Analytic approaches to human cognition (pp. 331-350). Amsterdam: Elsevier, North-Holland.

Weeks, D. J., \& Proctor, R. W. (1990). Salient-features coding in the translation between orthogonal stimulus-response dimensions. Journal of Experimental Psychology: General, 119, 355-366.

WeEks, D. J., Proctor, R. W., \& BeyaK, B. (1995). Stimulus-response compatibility for vertically oriented stimuli and horizontally oriented responses: Evidence for spatial coding. Quarterly Journal of Experimental Psychology, 48A, 367-383.

ZoRZI, M., \& UMILTÀ, C. (1995). A computational model of the Simon effect. Psychological Research, 58, 193-205.

(Manuscript received February 10, 2003; revision accepted for publication November 1, 2003.) 